\title{
Erdheim-Chester disease and slowly progressive cerebellar dysfunction
}

Toshiyuki Fukazawa, Eri Tsukishima, Hidenao Sasaki, Koji Hamada, Takeshi Hamada, Kunion Tashiro

\begin{abstract}
A 59 year old woman developed pronounced thirst, increased water intake, and increased urinary output followed by slowly progressive cerebellar symptoms. Brain MRI showed abnormal hyperintensity on $T 2$ weighted studies in the region of both dentate nuclei without atrophy of the cerebellum or the brainstem. A ${ }^{99 m}$ TC diphosphonate bone scan showed bone lesions in the distal parts of both femurs as well as distal and proximal parts of both tibias. The diagnosis of Erdheim-Chester disease was made by bone biopsy. This is the first case of Erdheim-Chester disease presenting as a slowly progressive cerebellar syndrome and diabetes insipidus, and also showing high signal lesions in deep cerebellar nuclei on MRI. Skeletal surveys are indicated for patients with otherwise unexplained slowly progressive cerebellar symptoms.
\end{abstract}

$(尹$ Neurol Neurosurg Psychiatry 1995;58:238-240)

Keywords: Erdheim-Chester disease; histiocytosis; cerebellar dysfunction

Erdheim-Chester disease is a rare histiocytosis of unknown aetiology with distinctive pathological and radiographic features characterised by bilateral, symmetric sclerosis of diaphyses and metaphyses of the long tubular bones and infiltration of foamy, lipid laden histiocytes. ${ }^{1}$ The disease seems to be a multisystemic histiocytic disorder, and retroperitoneal, cardiac, and pulmonary involvement have been noted in most cases. The most common site for intracranial accumulation of histiocytes seems to be the retro-orbital space with resultant proptosis or diplopia. Other neurological manifestations are extremely unusual. We present what may be the first reported case of Erdheim-Chester disease with slowly progressive cerebellar symptoms, diabetes insipidus, and high signal lesions in deep cerebellar nuclei on MRI.

\section{Case report}

A 59 year old woman developed pronounced thirst, increased water intake, and increased urinary output in April 1983, and was diagnosed as having idiopathic diabetes insipidus by a water deprivation test at another hospital. The symptoms of diabetes insipidus disappeared spontaneously within a year and she was in good health until October 1986. Then she developed a low grade fever, lower abdominal pain, and decreased urinary output. She was referred to a urologist and was diagnosed as having idiopathic retroperitoneal fibrosis. Ureterolysis was performed in May 1989 and the urinary symptoms disappeared. She developed an unsteady gait and dysarthria after the operation. She was admitted to Hokuyukai Neurology Hospital in October 1989. There was no family history of neurological disease and no alcohol or drug misuse. Examination showed a well nourished woman with neither exophthalmos nor bony tenderness. There was a xanthoma on the left upper eyelid but no tendon swelling. Cerebellar dysarthria with defective smooth pursuit eye movements was noted. Strength was normal but muscle tone was generally decreased. Sensory examination showed normal results except mild involvement of vibratory sensation in the lower extremities.

There was mild gait ataxia without dysmetria in all extremities. Laboratory tests of adrenal, thyroid, parathyroid, renal, hepatic, and haematological function, including alkaline phosphatase, showed normal results. The serum vitamin E concentration was within normal limits. The total serum cholesterol concentration was $265 \mathrm{mg} / \mathrm{dl}$ (normal $130-230 \mathrm{mg} / \mathrm{dl}$ ), and triglyceride was 161 $\mathrm{mg} / \mathrm{dl}$ (normal 40-150 mg/dl). Examination of CSF showed it to be normal. There was no definitive abnormality on brain CT, but MRI showed abnormal hyperintensity on T2 weighted studies in both dentate nuclear regions (fig 1). She was discharged with a tentative diagnosis of sporadic spinocerebellar degeneration, and the ataxia and dysarthria slowly worsened thereafter. In January 1993 she developed increased water intake and increased urinary output after operative treatment for cholelithiasis. The presence of diabetes insipidus with a satisfactory response to vasopressin was established by a water deprivation test. She was readmitted to Hokuyukai Neurology Hospital in June 1993. Examination showed a horizontal gaze evoked nystagmus and defective pursuit eye 
Figure 1 Axial T2 weighted MRI (TR = 3000, $T E=90$ ) showing abnormal hyperintensity in both dentate nuclei.

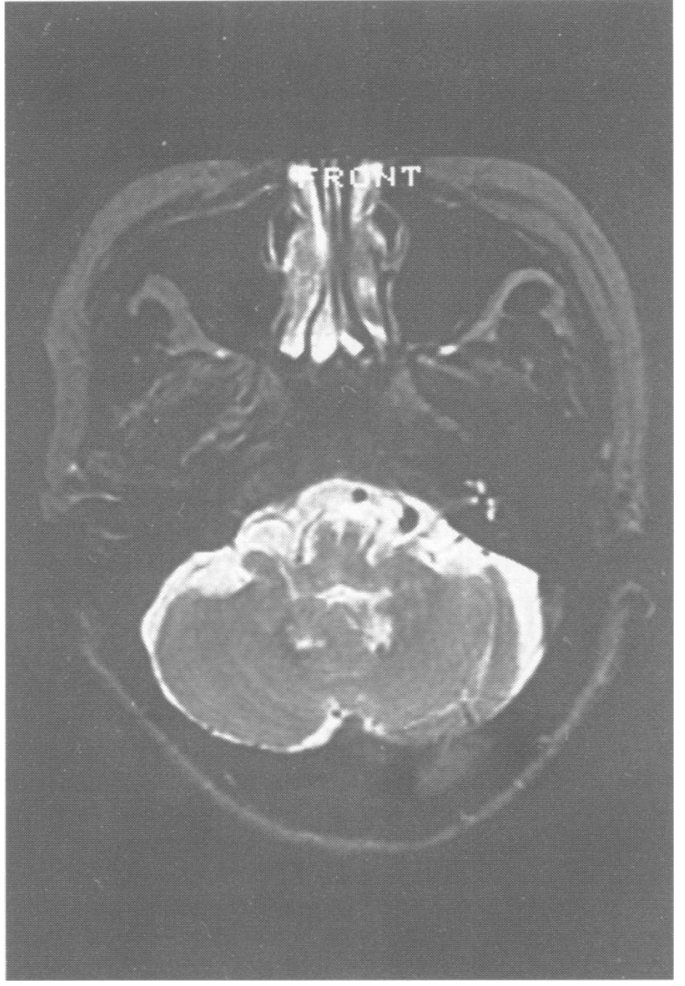

movement as well as a cerebellar dysarthria. Strength was normal but increased muscle tone of both upper extremities and bilateral Babinski signs were present. Dysmetria in all limbs was evident, and she could not stand by herself due to pronounced ataxia. Sensory examination results were normal except for mildly diminished vibratory sensation in the lower limbs. The results of laboratory tests and neuroimaging studies were essentially the same as those performed at the first admission. Cerebellar dysfunction with diabetes insipidus led to concern about histiocytosis and a series of skeletal surveys were performed. Radiographs showed mild, patchy sclerotic lesions in the distal part of the right

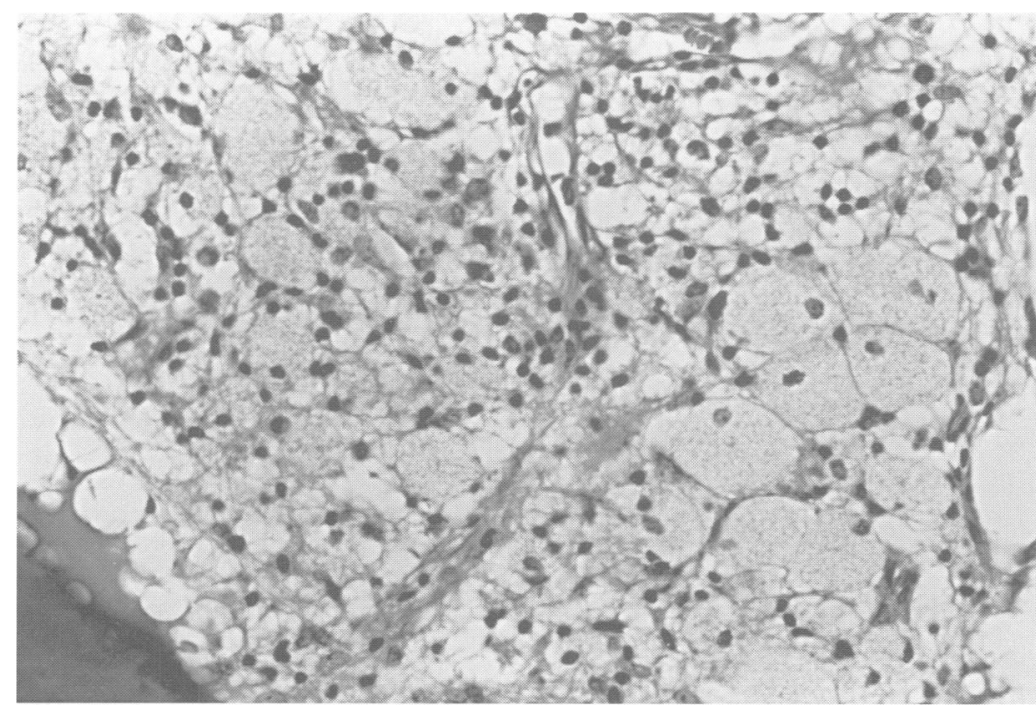

Figure 3 Lesion of the right tibia showing nests of aggregated foamy histiocytes characteristic of Erdheim-Chester disease. There was no granulomatous response and the inflammatory response was minimal (haematoxylin and eosin, originally $\times 100$ ).

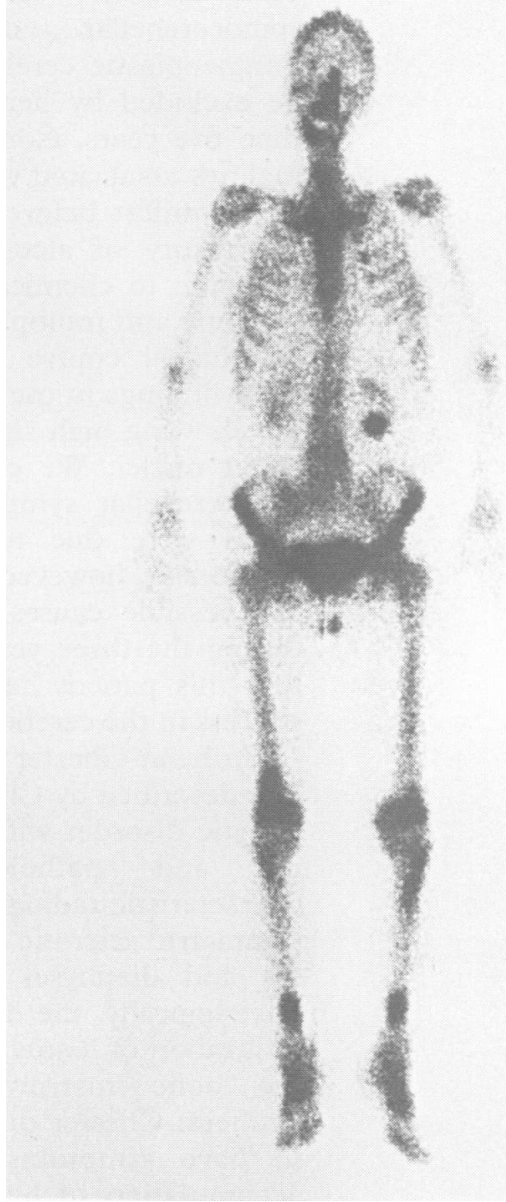

Bone scintigraphy with ${ }^{99 m} T c$ diphosphonate showing symmetrically increased uptake in the distal parts of both femurs, as well as the distal and proximal parts of both tibias. Focal uptake in the right mandible was compatible with dental disease.

femur and proximal parts of both tibias. A ${ }^{99 \mathrm{~m}} \mathrm{Tc}$ diphosphonate bone scan showed increased uptake in the distal parts of both femurs, as well as distal and proximal parts of both tibias (fig 2). Bone biopsies of the right femoral and tibial lesions were performed. Histological examination disclosed aggregated foamy histiocytes in the bone marrow (fig 3). There was no granulomatous response and inflammatory responses were minimal. The diagnosis of Erdheim-Chester disease was based on radiological and pathological findings. Ataxia has worsened very slowly and she has been confined to a wheelchair since January 1994. In the absence of definitive efficacy and with the possibility of adverse effects she has been reluctant to initiate either radiation therapy or chemotherapy.

\section{Discussion}

Our patient presented with symptoms of diabetes insipidus followed by slowly progressive cerebellar dysfunction. She also had radiographically and histologically characteristic findings of Erdheim-Chester disease in the bone. Various different processes can cause progressive ataxia. The absence of atrophy of either the cerebellum or the brainstem on neuroimaging studies and the presence of dia- 
betes insipidus are extremely atypical for spinocerebellar degenerative disorders. Paraneoplastic cerebellar degeneration could be excluded by her clinical course of more than five years. Congenital metabolic abnormalities associated with cerebellar ataxia usually manifest before adolescence. There was no history of alcoholism, drug misuse, or exposure to chemical agents. Posterior fossa tumours and multiple sclerosis were excluded by clinical course and neuroimaging. The MRI findings in our patient were characteristic, showing high signal lesions in deep cerebellar nuclei. We could not clarify whether the cerebellar symptoms and MRI appearances were due to histiocytic infiltration. There was, however, no evidence to indicate the possible causes of cerebellar symptoms during the three year follow up. We believe that this patient had the same pathological process in the cerebellum as in bone.

Erdheim-Chester disease is a rare disorder first described by Chester in $1930 .^{1}$ It is a histiocytic disorder with distinctive radiographical and pathological features. The characteristic radiographic finding is bilateral, symmetric sclerotic changes in the metaphyses and diaphyses of long tubular bones. Histologically, the disease is characterised by infiltration of foamy, lipid laden histiocytes in the bone marrow. Eight patients with Erdheim-Chester disease have been reported to have symptoms related to intracranial accumulation of histiocytes..$^{2-8}$ Seven of the eight patients had retro-orbital masses.-7 Proptosis with or without diplopia was found in six patients, ${ }^{235-7}$ and optic nerve involvement was also present in two patients. ${ }^{26}$ Two of seven patients with retro-orbital masses had diabetes insipidus as well as orbital manifestations. ${ }^{24}$ Two of eight patients had multiple lesions in the brain. ${ }^{78}$ Our patient developed slowly progressive cerebellar dysfunctions accompanied by diabetes insipidus, and MRI showed abnormal areas of hyperintensity on T2 weighted studies in the bilateral dentate nuclei. The hypothalamus or posterior pituitary gland may also have been involved due to infiltration of histiocytes with resultant diabetes insipidus, although no such lesions were found on imaging studies. Some cases with another form of histiocytosismultifocal eosinophilic granuloma-were reported to have presented as slowly progressive cerebellar dysfunction with various neuroimaging findings..$^{912}$ To our knowledge, however, this is the first reported case of
Erdheim-Chester disease with such clinical and radiological findings. Pathologically, several authors believe that there is little or no difference between multifocal eosinophilic granuloma and Erdheim-Chester disease. The preponderance of lipid laden histiocytes or foamy cells can, however, distinguish Erdheim-Chester disease from multifocal eosinophilic granuloma, in which there is a proliferation of mature histiocytes and eosinophils. Furthermore, bilateral metaphyseal sclerotic lesions of the long bones with epiphyseal sparing are characteristic of Erdheim-Chester disease. Our patient had also been diagnosed as having idiopathic retroperitoneal fibrosis during her clinical course. Retroperitoneal histiocyte infiltration may have been present, but precise information is not available.

The treatment and the progression of the disease are poorly understood, but some patients were reported to show temporary improvement of symptoms after radiation therapy or chemotherapy. Thus a skeletal survey is indicated for undiagnosed patients with cerebellar symptoms.

We are very grateful to Dr Naoki Takeda, of the Department of Orthopaedic Surgery and to Takayuki Nojima of the Department of Pathology, Hokkaido University School of Medicine for valuable assistance and comments.

1 Chester W. Ueber lipoidgranulomatose. Virchows Arch Pathol Anat Physiol 1930;279:561-602.

2 Alper MG, Zimmerman LE, La Piana FG. Orbital maniestations of Erdheim-Chester disease. Transactions of the Ophthalmological Society of America 1983;81:64-85.

3 Palmer FJ, Talley NJ. Erdheim-Chester disease with bilateral exophthalmus and liver cell adenoma. Australas
Radiol 1984;28:305-10.

4 Miller RL, Sheeler LR, Bauer TW, Bukowski RM. Erdheim-Chester disease. Case report and review of the literature. $A m \mathcal{F}$ Med 1986;80:1230-6.

5 Rozenberg I, Wechsler J, Koenig F, et al. ErdheimChester disease presenting as malignant exophthalmos. BrF Radiol 1986;59:173-7.

6 Sandrock D, Merino MJ, Schefflknecht BHB, Neumann RD. Scintigraphic findings and follow up in ErdheimChester disease. Eur f Nucl Med 1990;16:55-60.

7 Tien RD, Brasch RC, Jackson DE, Dillon WP. Cerebral Erdheim-Chester disease: Persistent enhancement with Gd-DTPA on MR images. Radiology 1989;172:791-2.

8 Smith ME, Katz DA, Harris JO, Frank JA, Kufta CV, McFarlin DE. Systemic histiocytosis presenting as multiple sclerosis. Ann Neurol 1993;33:549-54.

9 Braunstein GD, Whitaker JN, Kohler PO. Cerebellar dysfunction in Hand-Schuller-Christian disease. Arch Intern Med 1973;132:387-90.

10 Adornato BT, Eil C, Head GL, Loriaux DL. Cerebellar involvement in multifocal eosinophilic granuloma: Demonstration by computed tomographic scanning. Ann Neurol 1980;7:125-9.

11 Beard W, Foster B, Kepes JJ, Guillan RA. Xanthomatosis of the central nervous system. Clinical and pathological observations of a case with a posterior fossa syndrome. Neurology 1970;20:305-14.

12 Fukazawa $T$, Yanagihara $T$, Hamada $K$, Hamada $T$. Multifocal eosinophilic granuloma presenting as progressive brainstem and cerebellar dysf 